\title{
An improved water-filled impedance tube
}

\author{
Preston S. Wilson, Ronald A. Roy and William M. Carey \\ Department of Aerospace and Mechanical Engineering, Boston University \\ 110 Cummington Street, Room 101, Boston, Massachusetts 02215
}

Received:

Running title: An improved water-filled impedance tube

Short title: Water-filled impedance tube 


\section{ABSTRACT}

A water-filled impedance tube capable of improved measurement accuracy and precision is reported. The measurement instrument employs a variation of the standardized two-sensor transfer function technique. Performance improvements were achieved through minimization of elastic waveguide effects and through the use of sound-hard wall-mounted acoustic pressure sensors. Acoustic propagation inside the water-filled impedance tube was found to be well described by a plane wave model, which is a necessary condition for the technique. Measurements of the impedance of a pressure-release terminated transmission line, and the reflection coefficient from a water/air interface were used to verify the system.

PACS numbers: 43.58.Bh, 43.30.Xm, 43.30.Jx 


\section{INTRODUCTION}

Impedance tubes are commonly used to measure the acoustic properties of materials and structures (wind instruments, acoustical filters, etc.) with air or other gases as the host medium. The technology is mature, as evidenced by an extensive literature (see reviews by Beranek $^{1}$ and Dalmont ${ }^{2}$ ), the availability of standardized techniques, ${ }^{3,4}$ techniques for specialized cases, ${ }^{5,6}$ and the availability of commercially produced impedance tubes. Despite this maturity, measurement of impedance still requires great care and very careful control of the measurement parameters, such as system temperature and sensor position. ${ }^{2}$ Absolute calibration of impedance tube measurements remains difficult. There is currently no accepted material for use as a standard reference. ${ }^{3}$ Measurement precision (repeatability) can be reported, but in most cases, accuracy is estimated or inferred by comparison to theory or to the results of other measurement techniques.

The need for acoustic properties measurement also exists for materials or structures with water as the host medium, but there are two additional barriers to the realization of a waterfilled impedance tube. The first is the minimization of elastic waveguide effects which result from the coupling between the fill-liquid and the tube walls. The second is the measurement of acoustic pressure without perturbation of the field within the tube and absent of any signal corruption from wall motion.

There have been a number of water-filled impedance tube techniques and measurements reported in the literature. These include pulse tubes, ${ }^{7,8}$ active cancellation techniques, ${ }^{9,10}$ and two-sensor transfer function techniques. ${ }^{11,12}$ Although frequency independent, the water/air interface has been used in two of these works for measurement validation. ${ }^{7,11}$ It approximates a pressure-release surface with a reflection coefficient $R=-1=1 \angle 180^{\circ}$. Using a pulse tube technique, and reporting only the phase of the complex reflection coefficient, Kuhl et al. ${ }^{7}$ reported a maximum deviation of approximately $10^{\circ}$ degrees from the expected value of $180^{\circ}$ degrees. Adapting the standardized two-sensor transfer function technique for use in water, Corbett ${ }^{11}$ reported measurements of reflection coefficient magnitude and phase that deviated from the expected values by as much as $10 \%$ and 30 degrees, respectively.

Reported here is a water-filled impedance tube instrument, which utilizes the two-sensorthree-calibration technique ${ }^{13}$ and is capable of improved accuracy and precision. Although the present instrument operates between 5 and $9 \mathrm{kHz}$, the basic technique is not limited 
to such a small range of operation. The two-sensor three-calibration technique is reviewed in Sec. II. Performance improvements were achieved through minimization of elastic waveguide effects, discussed in Sec. III and through use of a non-intrusive wall-mounted acoustic pressure sensor, discussed in Sec. IV. For the two-sensor method to succeed, the acoustic waves travelling within the impedance tube, specifically between the sample and the acoustic sensors, must be plane waves to a high degree of approximation. Experiments used to validate the plane wave behavior of the waveguide used in the impedance tube are presented in Sec. V. In Sec. VI, the impedance tube instrument is described, the performance of the system is reported, a comparison to other systems is made, and the results of a measurement error analysis are presented.

\section{METHOD OF IMPEDANCE MEASUREMENT}

The standard two-sensor method ${ }^{3}$ requires plane wave propagation within a rigid tube and relies on the ability to non-invasively measure acoustic pressure at two positions in the tube. Using the measurement scheme illustrated in Fig. 1 and excitation of the source with random noise, the transfer function is measured between the two acoustic pressure sensors. This transfer function is a complex quantity composed of the amplitude ratio and phase difference between the acoustic pressure measured at positions 2 and 1 . From the transfer function measurement, one can calculate either the complex reflection coefficient or the normal specific acoustic impedance at the interface. ${ }^{3}$ In the standard method, a calibration procedure must also be performed using a sensor switching technique and a strongly absorbing termination. Switching wall-mounted sensors back and forth between the two measurement positions (with the required precision) is impractical with a water-filled tube, as is a strongly absorbing termination. Therefore, the two-sensor three-calibration method $^{13}$ is used, in which no sensor switching or absorptive termination is required. Instead, calibration is achieved through the use of three reference terminations with known impedance values. Otherwise, the method is equivalent to the standard method.

An open-ended, water-filled tube of the same inner diameter and wall thickness as the impedance tube is used as the reference termination. The water height is varied to achieve terminations of three different lengths, which are selected such that resonance and antiresonance are avoided, thereby greatly reducing the importance of a detailed theoretical 
description of the loss mechanisms. ${ }^{13}$ This requirement limits the usable frequency range for any set of three reference terminations to about two octaves. Multiple sets of reference terminations (and hydrophone spacings) are required in order to realize an increased frequency range.

The procedure and theoretical formulation from Ref. 13 for measurement of impedance using the two-sensor-three-calibration technique is summarized below. A complete derivation is given in Ref. 13. The frequency is kept below cut-off for the first higher order mode, such that only plane waves travel within the tube. The calibration is performed first, with the reference terminations and the impedance tube filled with distilled water with known density $\rho_{1}$ and sound speed $c_{1}$. One measures the transfer function $y^{(\mathrm{i})}$ for each of the three reference terminations $Z^{(\mathrm{i})}$. Subsequently, measurement of the transfer function $y$ for any unknown termination yields its impedance $z$ in terms of the three calibration transfer functions and $\rho_{1} c_{1},{ }^{13}$

$$
\frac{z}{\rho_{1} c_{1}}=\frac{A+B+C}{D+E+F},
$$

where

$$
\begin{aligned}
& A=Z^{(1)} Z^{(3)}\left(y^{(1)}-y^{(3)}\right)\left(y-y^{(2)}\right) \\
& B=Z^{(1)} Z^{(2)}\left(y^{(2)}-y^{(1)}\right)\left(y-y^{(3)}\right) \\
& C=Z^{(3)} Z^{(2)}\left(y^{(3)}-y^{(2)}\right)\left(y-y^{(1)}\right) \\
& D=Z^{(1)}\left(y^{(3)}-y^{(2)}\right)\left(y^{(1)}-y\right) \\
& E=Z^{(2)}\left(y^{(1)}-y^{(3)}\right)\left(y^{(2)}-y\right) \\
& F=Z^{(3)}\left(y^{(2)}-y^{(1)}\right)\left(y^{(3)}-y\right) .
\end{aligned}
$$

The reference terminations are modeled as pressure-release-terminated transmission lines, with dimensionless impedances $Z^{(1)}, Z^{(2)}$, and $Z^{(3)}$, each given by

$$
Z^{(\mathrm{i})}=z^{(\mathrm{i})} / \rho_{1} c_{1}=i \tan k d_{\mathrm{i}},
$$

where $k=\omega / c_{1}$, the length of the termination is $d_{\mathrm{i}}$, and a time dependence $\exp (i \omega t)$ has been assumed. It is reiterated that if the calibration lengths $d_{\mathrm{i}}$ are chosen such that resonance and antiresonance of Eq. (2) is avoided, loss mechanisms can be neglected and a real wave number $k=\omega / c_{1}$ is sufficient. This is demonstrated in Sec. VI. 


\section{ELASTIC WAVEGUIDE EFFECTS}

The plane wave assumption used in the derivation of Eqs. (1) and (2) is easily satisfied with an air-filled impedance tube at low enough frequencies, because the tube walls are effectively rigid. For acoustic propagation within a water-filled impedance tube with steel walls, a number of elastic waveguide effects can arise, including a dispersive sound speed, radial particle displacement, wavefront curvature, and the existence of at least one higherorder mode at all frequencies.

Del Grosso ${ }^{14}$ obtained particle displacement field equations (Eqs. (23), Ref. 14) and a dispersion relation (Eq. (24), Ref. 14) for the axisymmetric modes of an inviscid-liquid-filled cylindrical tube with arbitrary-thickness elastic walls, where the outermost radial boundary condition was approximated as pressure release. These equations were used to evaluate a variety of candidate waveguide dimensions, using stainless steel as the wall material. The calculations indicate that a wall-thickness to inner-radius ratio close to unity results in less than $1 \%$ dispersion in the lowest-order mode (labeled ET0 by Del Grosso), up to approximately the rigid waveguide cutoff frequency. The ET0 mode exhibits nearly plane wavefronts and little radial particle motion. The next higher-order mode (ET1) persists down to zero-frequency but it displays about 14 times more wavefront curvature and about twenty times more radial particle motion than ET0. Based on these results, it appears that waves excited by a piston source isolated from the wall of the cylinder should couple well into ET0 and poorly into ET1, leaving only plane-like waves propagating in the waveguide.

Although Del Grosso's results have been experimentally verified for a number of cases, ${ }^{15}$ experiments were conducted to confirm the theory for the parameters of interest here. A thick-walled stainless steel cylindrical tube was employed with dimensions and material properties shown in Table I. An aluminum piston driven by a mass-loaded piezoelectric element (shown in Fig. 2) was fitted to the bottom of the tube, which was positioned vertically and filled with distilled and degassed water. Time-of-flight measurements were conducted inside the tube using a miniature hydrophone (Brüel and Kjær Model 8103) positioned just under the surface of the water at the open end of the tube. Four cycle sinusoidal pulses were generated and these propagated back and forth between the source and the open end. The hydrophone signal was band pass filtered and recorded using a digital oscilloscope. The pulses were sufficiently separated in time and the time between 
pulses was extracted using autocorrelation analysis. Results obtained at several frequencies are presented in Fig. 3 and compare favorably with Del Grosso's Eq. (24). The predicted and measured dispersion from $3 \mathrm{kHz}$ to $15 \mathrm{kHz}$ is less than $0.5 \%$, and the speeds are just a few percent below the intrinsic medium sound speed.

Wavefront curvature was measured by scanning a $1 \mathrm{~mm}$ diameter needle hydrophone across the top of the tube, $1 \mathrm{~cm}$ below the water surface. Continuous wave excitation was used and rms pressure measurements, made with a digital oscilloscope, were recorded as a function of position. The radial pressure profiles exhibited a concave-up curvature. Deviation between the acoustic pressure at the center of the tube and at the wall was $1 \%$ at $5 \mathrm{kHz}, 2 \%$ at $10 \mathrm{kHz}$ and $5 \%$ at $15 \mathrm{kHz}$, in qualitative agreement with predictions made using Del Grosso's Eqs. (23).

These measurements confirm that the field inside the waveguide is not truly plane. A small amount of dispersion and wavefront curvature is present. It is shown in Sec. V that despite this deviation from plane wave behavior, a plane wave model is sufficient to describe propagation inside this waveguide.

\section{ELIMINATION OF PERTURBATIONS CAUSED BY SENSORS}

Corbett $^{11}$ reported that hydrophones deployed within a water-filled impedance tube could perturb the acoustic field and along with sensor location uncertainty, could contribute significantly to impedance measurement error. Wall-mounted sensors could be less invasive and positioned with greater accuracy, but tube wall motion prevented their use. Calculations using Del Grosso's Eqs. (23) and (24) indicated that for the waveguide parameters given in Table I, both radial and longitudinal particle motion is present in the tube wall. A wallmounted hydrophone with reduced wall coupling in both directions is needed. However, a high impedance in the radial direction must be maintained since the wall-mounted sensor must not appear as an acoustically soft region in the wall.

This was achieved using a lead-zirconate-titanate (PZT) disc transducer mounted in a cylindrical steel housing, as shown in Fig. 4. The two parts of the housing are threaded together and compress the mica/PZT/mica layered stack between the steel window and the base of the housing, which results in sensitivity to acoustic pressure at the center of the steel window. The mica layers electrically isolate the PZT element and help to decouple shearing 
motion between the sensor housing and the PZT element, yet stiffness in the radial direction is maintained. Isolation from wall motion is primarily provided by the two rubber o-rings. Despite being free to move in the radial direction in response to a constant radial force, the

density and stiffness of the hydrophone results in a high acoustical input impedance. Some clearance must be present between the sensor and the mounting hole, so that the o-rings provide isolation. However, too much clearance results in acoustic energy loss. A mounting hole with a diameter $0.4 \%-0.8 \%$ larger than the hydrophone's diameter was found to be sufficient.

An experiment was conducted to verify that the presence of these hydrophones did not significantly perturb the acoustic field inside the impedance tube. Using the same arrangement described in Sec. III, the source was excited with a broadband signal and a spectrum analyzer was used to measure the acoustic pressure as a function of frequency. Spectra were measured in the tube before the mounting holes were drilled and again after the hydrophones were installed. The average deviation between the before and after spectra was approximately $0.5 \mathrm{~dB}$ in the operating range of the impedance tube, $5-9 \mathrm{kHz}$.

Air trapped in the clearance gap and the in the o-ring grooves can also introduce acoustic energy loss and alter the boundary conditions at tube wall. It is difficult to prevent this unless the insertion of the hydrophones into the mounting holes is done underwater. If this is not possible, wetting of the phones in degassed water prior to insertion is helpful. Subsequent filling of the impedance tube with degassed water will then allow for the dissolution of any trapped air. In practice, this can take a number of hours but the effect of trapped air is easily detectable. With a highly reflective water/air interface terminating the impedance tube, the measured transfer function between the hydrophones will exhibit sharp peaks and nulls in a properly functioning and air-free system. These extrema are due to nulls in the pressure field which occur at integer multiples of a half wavelength from the top of the tube. The presence of even minute air bubbles will change the effective sound speed and induce acoustic energy loss and thereby shift the frequency and reduce the sharpness of these extrema.

\section{VERIFICATION OF PLANE WAVE PROPAGATION}

Two experiments were conducted to determine if a plane wave model was sufficient to describe acoustic propagation in the impedance tube. Furthermore, propagation measurements 
serve as an additional check that the wall-mounted sensors do not perturb the field.

In the first experiment, the frequency response of the open water-filled tube was measured, as described in Sec. IV and the resonance frequencies were obtained. A piston driven by an electromagnetic shaker was used as a source in this experiment. Modelling it as a simple harmonic oscillator with constant force input, suspension stiffness $s$ and piston mass $m$, resonance of the combined source/tube system occurs when ${ }^{17}$

$$
\omega m-s / \omega+\rho_{1} c_{\mathrm{eff}} S \tan k_{\mathrm{eff}} L=0,
$$

where $\omega$ is the angular frequency, $S$ is the cross-sectional area, $L$ is the length of the tube and $k_{\text {eff }}=\omega / c_{\text {eff }}$. The phase speed predicted by Del Grosso's Eq. (24) averaged across the experimental frequency range was used as the effective sound speed $c_{\text {eff }}$ in the tube, which was $1463 \mathrm{~m} / \mathrm{s}$ for the experimental temperature of $25.5^{\circ} \mathrm{C}$. Equation (3) was solved numerically, using the parameters from Table I, and previously measured ${ }^{16}$ source parameters $m=0.187$ $\mathrm{kg}$ and $s=3500 \mathrm{~N} / \mathrm{m}$. The results are shown in Fig. 5. Once the dynamics of the source are considered, a plane wave model with an effective sound speed based on the properties of the elastic waveguide is sufficient to make accurate resonance predictions.

The experiment was repeated at a fifty-one different positions down the center of the tube, which yielded measurements of the standing wave pattern at a number of frequencies. The pressure amplitude of standing plane waves in a tube with a velocity boundary condition at $x=0$ and vanishing pressure at $x=L$ is given by

$$
p(x)=A_{0} \frac{\sin \left[k_{\mathrm{eff}}(L-x)\right]}{\cos k_{\mathrm{eff}} L},
$$

where $A_{0}$ is an amplitude factor proportional to piston velocity, and $k_{\text {eff }}=\omega / c_{\text {eff }}-i \alpha_{0}$. Agreement between measurement and Eq. (4) is seen in Fig. 6, where the measured data have been normalized by the maximum pressure at each frequency, $A_{0}$ was set to unity, the predicted effective sound speed was $c_{\mathrm{eff}}=1450 \mathrm{~m} / \mathrm{s}$ at the experimental temperature of $20.6{ }^{\circ} \mathrm{C}$, and $\alpha_{0}=0.09 \mathrm{~m}^{-1}$ was obtained from the best fit between measurement and Eq. (4) at the pressure null near $x=0.4 \mathrm{~m}$ in the bottom frame of Fig. 6.

The experimental results shown in Figs. 5 and 6 indicate that a plane wave model, suitably modified by the use of an effective sound speed, is capable of describing the resonance frequencies and standing wave patterns of the current water-filled waveguide. There is a small amount of attenuation in the system but the imaginary part of the wave number is less than one-half percent of the real part above $5 \mathrm{kHz}$. 


\section{IMPEDANCE TUBE INSTRUMENT}

The length of the tube already described was appropriate for propagation studies but the effects of attenuation, already seen to be small, are further reduced by minimizing the tube length. Following tube length and sensor separation distance guidelines given in the ASTM standard document, ${ }^{3}$ an impedance tube and sample holder was constructed using tube radii nominally the same as those already discussed. Tube dimensions are presented in Fig. 7 and a schematic diagram of the instrumentation and supporting equipment appears in Fig. 8. The piezoelectric-driven piston source described in Fig. 2 was used but any pistontype source is sufficient, as long as it is decoupled from the tube wall and excites plane waves with a signal to noise ratio greater than $10 \mathrm{~dB} .{ }^{3}$ Note that increasing the sensor separation distance $s$ lowers the minimum usable frequency of the instrument and decreasing the inner radius of the tube increases the maximum usable frequency. ${ }^{3,13}$

\section{A. Measurement procedure}

The first step in the procedure involves measurement of the effective propagation speed in the tube. System calibration is sensitive to small changes in the speed of sound in the tube and therefore sensitive to temperature, which is monitored using a digital multi-meter and a thermocouple attached to the outside of the tube. Calibration and sound speed measurement must be repeated if the temperature of the tube changes by about $0.5^{\circ} \mathrm{C}$ but the higher heat capacity of water makes the water-filled impedance tube less sensitive to room temperature changes than an air-filled tube.

The tube is filled with distilled and degassed water, preferably several hours before operation. This insures temperature equilibrium and the dissolution of any air trapped between the sample holder and the tube, around the source piston, or around the hydrophones. The water level is obtained to approximately $0.05 \mathrm{~mm}$ accuracy by using the bifilar microscope to measure the distance from the water surface to the top of the tube. Signal generation and transfer function measurement is performed by a Hewlett-Packard 89410A signal analyzer. The sound source is driven with $5-9 \mathrm{kHz}$ periodic chirps or band-limited pseudorandom noise; chirps are useful for single short-time measurements and noise is useful for time-averaged measurements. The transfer function $y_{0}$ between the two wall-mounted hy- 
drophones is measured and from the position of nulls and peaks, the in-situ sound speed $c_{\text {eff }}$ can be calculated based on the water level.

The system is now ready for the three calibration measurements to be performed. The lengths 1.5, 2.5 and $3.5 \mathrm{~cm}$ are selected to avoid any extrema, as previously mentioned. The appropriate volume of water is removed by monitoring the milligram balance and a transfer function $\left(y^{(1)}, y^{(2)}, y^{(3)}\right)$ is measured for each length $\left(d_{1}, d_{2}, d_{3}\right)$. This completes the calibration procedure. The test material is installed and impedance of subsequent measurements are calculated using the three calibration measurements $\left(y^{(1)}, y^{(2)}, y^{(3)}\right)$ and Eq. (1). In order to minimize bias error in the transfer function measurements, the resolution bandwidth is maximized and a sufficient number of averages obtained. ${ }^{18}$ If a membrane is used to separate the sample from the water below the interface, the lengths of the calibration terminations must be set by removal of water from the top of the tube with a volumetric pipette.

The calibration can be verified efficiently by using the first transfer function measurement $y_{0}$ as a test case. This corresponds to a fourth length of fluid $d_{0}$, which can be considered a pressure release terminated transmission line, whose length is known from the microscope measurement.

\section{B. Verification of impedance tube system}

The pressure-release-terminated transmission line described by Eq. (2) has a complex, frequency dependent input impedance. Short lengths with no resonances or antiresonances are used as calibration terminations and losses are neglected. Longer transmission lines do contain extrema and serve as a difficult test for any impedance tube because their input impedances can vary over several orders of magnitude. Verification measurements are compared to a model in which propagation in the bulk fluid is still considered lossless but interaction with the walls will be accounted for using a complex wave number

$$
\widehat{k}=\frac{\omega}{c_{\mathrm{eff}}}+(1-i) \alpha_{\mathrm{walls}}
$$

The approximation $\omega / c_{\text {eff }} \gg \alpha_{\text {walls }}$ is assumed and thermal losses are considered negligible compared to viscous losses, which are characterized by ${ }^{19}$

$$
\alpha_{\mathrm{walls}}=\frac{1}{a} \sqrt{\frac{\mu \omega}{2 \rho_{1} c_{\mathrm{eff}}^{2}}},
$$


where $a$ is the inner radius of the tube and the viscosity of distilled water is taken to be $\mu=0.907 \times 10^{-3} \mathrm{~kg} /(\mathrm{m} \cdot \mathrm{s}), \rho_{1}=997 \mathrm{~kg} / \mathrm{m}^{3}, c_{\text {eff }}=1457 \mathrm{~m} / \mathrm{s}$, all at the experimental temperature of $24.0^{\circ} \mathrm{C}$.

Energy also leaves the system via the water/air interface at the open end of the sample holder. Approximating this as a piston in an infinite baffle and using Eq. (8.68b) in Ref. 17, one finds that in the $5-9 \mathrm{kHz}$ frequency range, the magnitude of the radiation impedance varies about the progressive plane wave value $(\rho c)_{\text {air }}$ and possesses an average value within $6 \%$ of $(\rho c)_{\text {air }}$. The sample holder was therefore modeled as layer of water terminated with an infinite length air-filled transmission line, with input impedance

$$
z=\rho_{1} c_{\mathrm{eff}} \frac{1+R}{1-R}
$$

where the three-medium reflection coefficient is ${ }^{17}$

$$
R=\frac{\left(1-r_{1} / r_{3}\right) \cos \widehat{k} d+i\left(r_{2} / r_{3}-r_{1} / r_{2}\right) \sin \widehat{k} d}{\left(1+r_{1} / r_{3}\right) \cos \widehat{k} d+i\left(r_{2} / r_{3}+r_{1} / r_{2}\right) \sin \widehat{k} d}
$$

Losses are neglected for the water below the sample holder and for the air outside, therefore $r_{1}=\rho_{1} c_{\text {eff }}$ and $r_{3}=(\rho c)_{\text {air }}=411 \mathrm{~Pa} \cdot \mathrm{s} / \mathrm{m}$ at $24.0{ }^{\circ} \mathrm{C}$. Losses are accounted for within the sample holder using $\widehat{k}$ and $r_{2}=\rho_{1} \frac{\omega}{\widehat{k}}$. The height of the water within the sample holder is $d$.

Two verification measurements are shown in Fig. 9 and compared with predictions given by Eqs. (5)-(8). Pseudo-random noise excitation was used, with a Hann window, a resolution bandwidth of $5 \mathrm{~Hz}$, and 50 averages.

To verify that the lossless description of the reference terminations used in the calibration was appropriate, the formulation given by Eq. (7) was compared to Eq. (2) for the three reference termination lengths and found to differ in magnitude by at most 8 parts in 10,000 and typically by less than 2 parts in 10,000. In addition, Eq. (7) was substituted for Eq. (2) and then Eq. (1) was recalculated for the $d=13.98 \mathrm{~cm}$ case. The difference between this and the measurement result shown in Fig 9, averaged across 5-9 kHz, was less than $0.0012 \mathrm{~dB}$. The greatest difference, which only occurred at few data points immediately surrounding the extrema, was less than $1 \mathrm{~dB}$.

The reflection coefficient of a water-air interface was used for further system verification. The impedance $z$ was measured from a water/air interface and converted to a reflection coefficient by using $R=\left(z-\rho_{1} c_{\text {eff }}\right) /\left(z+\rho_{1} c_{\text {eff }}\right)$. The result is shown in Fig. 10 and should be compared to the expected value of $R \simeq 1 \angle 180^{\circ}$. The increased scatter below $6 \mathrm{kHz}$ is 
due to reduction of signal-to-noise ratio caused by the limited frequency range of the source used in this system. This is most apparent in Fig. 10, but can also be seen in the phase measurement for the 14-cm case in Fig. 9.

In order to compare this reflection coefficient measurement to the two others that were found in the literature, ${ }^{7,11}$ phase and magnitude values were averaged across the $5-9 \mathrm{kHz}$ frequency range to obtain mean values, representing accuracy. Measurement range was used to create error bars, representing precision. The three are compared in Fig. 11. It is clear that for this particular case, the present impedance tube yields the most accurate and precise measurement.

The repeatability of the instrument was examined by obtaining 50 impedance measurements of the 14-cm length air-terminated transmission line over the course of 35 minutes. Each of the individual measurements was made as described for Fig. 9, except a periodic chirp was used as the excitation signal. It was found that the measured impedance was changing over time in a manner quantitatively consistent with a length change due to evaporation of water from the open end of the sample holder. This trend was removed and the standard deviation of the magnitude and phase of the impedance at each frequency bin was calculated and then averaged over the 5-9 kHz frequency range. The result, representing frequency-averaged repeatability, was $\pm 0.0035 \mathrm{~dB}$ re. $\rho_{1} c_{\text {eff }}$ in impedance magnitude and $\pm 0.027^{\circ}$ in impedance phase.

\section{Measurement error}

A number of authors have analyzed the sources of error in the two-sensor impedance tube measurement technique. ${ }^{18,20,21}$ There are two classes: errors associated with the measurement of transfer functions and errors associated with the other measured parameters (sensor position, length of the reference terminations, speed of sound within the impedance tube). Approximate analytical formulations exist for the prediction of the former class of error, which is proportional to the magnitude of the reflection coefficient. Highly reflective terminations, such as those used here, are subject to the maximum error. Direct numerical simulation is used for the later class. A theoretical analysis of error using these techniques was done for this impedance tube ${ }^{16}$ and the results are reported here, in terms of rms error in magnitude and phase of impedance, averaged across the 5-9 $\mathrm{kHz}$ frequency range. The 
approximate predicted impedance measurement error for a $14 \mathrm{~cm}$ long pressure release terminated transmission line is $0.190 \mathrm{~dB}$ in magnitude and 0.42 degrees in phase. Assuming that the theoretical curves shown in part (a) and (b) of Fig. 9 represent the true impedance, the actual measurement error was $0.28 \mathrm{~dB}$ in magnitude and 0.95 degrees in phase, which is in reasonable agreement with the predicted error.

\section{CONCLUSIONS}

The two-sensor-three-calibration impedance measurement technique has been implemented in a water-filled impedance tube. For the technique to be successful, propagation within the tube must be limited to plane waves and measurement of acoustic pressure must be achieved without perturbation the acoustic field within the tube. The first criterion was met by minimization of the elastic waveguide effects which are inherent in a liquid-filled tube. Using an elastic waveguide model for a cylindrical tube, inner and outer wall radii were found which minimized these effects over the frequency range of interest, 5-9 kHz. The second criterion was met by using a vibration isolated, wall-mounted acoustic pressure sensor, with a high acoustic input impedance that maintained the sound-hard boundary condition along the tube wall.

Using a water/air interface as a reference termination, the performance of the present water-filled impedance tube system was compared to the performance of earlier systems and increased measurement accuracy and precision was found. Although the present instrument was designed for a particular range of frequencies, tube radii and sensor positions could be modified for use at other frequencies.

\section{ACKNOWLEDGMENTS}

This work was supported by the U. S. Navy Office of Naval Research Ocean Acoustics Program and Boston University.

\section{REFERENCES}

${ }^{1}$ L. L. Beranek, Acoustical Measurements (Acoustical Society of America, Woodbury, NY, 1988), Chap. 7, pp. 294-353. 
${ }^{2}$ J. P. Dalmont, "Acoustic impedance measurement, Part I: A review," J. Sound. Vib. 243, 427-439 (2001).

${ }^{3}$ ASTM, "Standard test method for impedance and absorption of acoustical materials using a tube, two microphones, and a digital frequency analysis system," ASTM E 1050-98, American Society for Testing and Materials, West Conshohocken, PA (1998).

${ }^{4}$ ASTM, "Standard test method for impedance and absorption of acoustical materials by the impedance tube method," ASTM C 384-95, American Society for Testing and Materials, West Conshohocken, PA (1995).

${ }^{5}$ M. Åbom and H. Bodén, "Error analysis of two-microphone measurements in ducts with flow," J. Acoust. Soc. Am. 83, 2429-2438 (1988).

${ }^{6}$ Y. Champoux and M. R. Stinson, "Measurement of the characteristic impedance and propagation constant of materials having high flow resistivity," J. Acoust. Soc. Am. 90, 2182-2191 (1991).

${ }^{7}$ W. Kuhl, E. Meyer, H. Oberst, E. Skudrzyk, and K. Tamm, Sound Absorption and Sound Absorbers in Water (Dept. of the Navy, Bureau of Ships, Washington, DC, 1947), Vol. 1, Chap. IX, pp. 381-453.

${ }^{8}$ G. A. Sabin, "Acoustic-impedance measurements at high hydrostatic pressures," J. Acoust. Soc. Am. 40, 1345-1353 (1966).

${ }^{9}$ D. M. Kenney, "A short water-filled pulse tube for the measurement of the acoustic properties of materials at low frequencies," Technical Report No. NSWCCD-TR-97/029, Naval Surface Warfare Center Carderock Division, West Bethesda, Maryland (1997).

${ }^{10}$ J. C. Piquette and S. E. Forsythe, "Low-frequency echo-reduction and insertion-loss measurements from small passive-material samples under ocean environmental temperatures and hydrostatic pressures," J. Acoust. Soc. Am. 110, 1998-2006 (2001).

${ }^{11} \mathrm{~S}$. S. Corbett III, A Two-Hydrophone Technique for Measuring the Complex Reflectivity of Materials in Water-Filled Tubes (M. S. thesis, Pennsylvania State University, 1983).

${ }^{12}$ J. I. Dunlop, "Measurement of acoustic attenuation in marine sediments by impedance 
tube," J. Acoust. Soc. Am. 91, 460-469 (1992).

${ }^{13} \mathrm{~V}$. Gibiat and F. Laloë, "Acoustical impedance measurements by the two-microphonethree-calibration (TMTC) method," J. Acoust. Soc. Am. 88, 2533-2545 (1990).

${ }^{14}$ V. A. Del Grosso, "Analysis of multimode acoustic propagation in liquid cylinders with realistic boundary conditions-application to sound speed and absorption measurements," Acustica 24, 299-311 (1971).

${ }^{15}$ L. D. Lafleur and F. D. Shields, "Low-frequency propagation modes in a liquid-filled elastic tube waveguide," J. Acoust. Soc. Am. 97, 1435-1445 (1995).

${ }^{16}$ P. S. Wilson, Sound Propagation in Bubbly Liquids (Ph.D. dissertation, Boston University, 2002), pp. 62-145 and pp. 257-268.

${ }^{17}$ L. E. Kinsler, A. R. Frey, A. B. Coppens, and J. V. Sanders, Fundamentals of Acoustics, 3rd ed. (Wiley, New York, 1982), pp. 210-214 and p. 128.

${ }^{18}$ J. S. Bendat and A. G. Piersol, Engineering Applications of Correlation and Spectral Analysis, 2nd ed. (J. Wiley, New York, 1993), Sec. 5.2, pp. 113-124.

${ }^{19}$ A. D. Pierce, Acoustics: An Introduction to Its Physical Principles and Applications (Acoustical Society of America, Woodbury, NY, 1994), Sec. 10-5, pp. 531-534.

${ }^{20}$ A. F. Seybert and B. Soenarko, "Error analysis of spectral estimates with application to the measurement of acoustic parameters using random sound fields in ducts," J. Acoust. Soc. Am. 69, 1190-1199 (1981).

${ }^{21} \mathrm{H}$. Bodén and M. Åbom, "Influence of errors on the two-microphone method for measuring acoustic properties in ducts," J. Acoust. Soc. Am. 79, 541-549 (1986).

${ }^{22}$ ASM International, Metals Handbook, 9th ed. (ASM International, Materials Park, OH, 1989), Vol. 17, p. 235. 
TABLE I. Material and geometric parameters of the experimental waveguide discussed in Secs. III$\mathrm{V}$ are shown. The inner liquid was distilled water, with sound speed $c_{1}$ calculated from a temperature measurement and density $\rho_{1}$. The tube wall material was grade $304 \mathrm{~L}$ stainless steel, with longitudinal and transverse sound speeds $c_{l}$ and $c_{t}$ respectively, ${ }^{22}$ and density $\rho_{\mathrm{s}}$ measured by volumetric displacement of a small sample. Tube dimensions are inner radius $a$, outer radius $b$, and length $L$.

\begin{tabular}{lccc}
\hline \hline \multicolumn{2}{c}{ sound speeds } & & \\
\multicolumn{1}{c}{ water } & 304L s.s. & densities & dimensions \\
\hline$c_{1}=1491 \mathrm{~m} / \mathrm{s}$ & $c_{l}=5640 \mathrm{~m} / \mathrm{s}$ & $\rho_{1}=998 \mathrm{~kg} / \mathrm{m}^{3}$ & $a=2.54 \mathrm{~cm}$ \\
& $c_{t}=3070 \mathrm{~m} / \mathrm{s}$ & $\rho_{\mathrm{s}}=7970 \mathrm{~kg} / \mathrm{m}^{3}$ & $b=5.08 \mathrm{~cm}$ \\
& & $L=150.2 \mathrm{~cm}$ \\
\hline \hline
\end{tabular}




\section{LIST OF FIGURES}

1 A schematic diagram of the impedance tube system is shown. A cylindrical tube has a piston-source at the bottom. Acoustic pressure sensors are wall mounted at positions labeled 1 and 2. Below the interface, the tube is filled with distilled water with known properties $\rho_{1} c_{1}$. Above the interface, a material with unknown properties $\rho_{2} c_{2}$ fills the tube to a height $d_{\mathrm{i}}$. Sensor 1 is positioned a distance $l$ from the interface and sensor separation distance is $s$. Coordinate axes indicate the longitudinal $x$ and the radial $r$ directions. . . .

2 A schematic diagram of the sound source is shown in a cut-away view. A Tonpilz-type transducer is enclosed and mounted in an air-filled stainless steel housing. The active elements, labeled $(*)$, are de-coupled from the housing using rubber o-rings. The housing is sealed to the impedance tube using another o-ring in the flange (not shown). The electrical wiring (not shown) exits the housing through a water-proof cable pass-through. . . . . . . . .

3 Measured waveguide phase speed is compared to Eq. (24) from Del Grosso ${ }^{14}$ for the plane-like ET0 mode. The values are normalized by $c_{1}$, the intrinsic sound speed in water for the experimental temperature. Error bars due to $\pm 0.5 \mathrm{~mm}$ uncertainty in waveguide length and $2.5 \mu$ s time resolution are shown. 22 (a) A schematic diagram of the wall-mounted hydrophone is shown in a cutaway view. The radial direction is indicated with an arrow and the letter $r$. (b) A more detailed schematic of the hydrophone is shown in exploded view. The dimensions of the disk-shaped PZT element are: $6.35 \mathrm{~mm}$ diameter $\times$ $2.54 \mathrm{~mm}$ thick. A shielded cable (not shown) is potted into the cable compartment. Mechanical drawings and additional details are available. ${ }^{16} \mathrm{Hy}-$ drophone sensitivity is $-233 \pm 3 \mathrm{~dB}$ re $1 \mathrm{~V} / \mu \mathrm{Pa}$ from $1-20 \mathrm{kHz}$. . . . . .

5 Measured resonance frequencies of the open, water-filled tube are shown and compared to the model. The solid line is drawn through the resonances predicted by Eq. (3) for the combined source/tube system. The dashed line is drawn through the resonances predicted by Eq. (3) with $m=s=0$, representing the acoustics of the tube with no source. . . . . . . . . . . . 24 
6 The standing wave pattern within the impedance tube at four frequencies: Measurement (open circles) and Eq. (4) (solid line). . . . . . . . . . . 25

7 The dimensions of the impedance tube and sample holder are shown. The material is 304L stainless steel. The two sections are attached using flanges. If needed, a thin mylar sheet can be fitted between the sample holder and the tube, to separate the sample from the water below the interface. . . . . . . 26

8 The impedance tube system, including support equipment and instrumentation is shown in a schematic diagram. Signals from the acoustic pressure sensors (labeled $1 \& 2$ ) are conditioned with a charge amplifier and recorded with a Hewlett-Packard 89410A signal analyzer, which also generates the excitation signal. A power amplifier increases the voltage of the excitation signal. A bifilar microscope is used to measure the height of the fluid column relative to the top of the tube. Additional details are given in the text. . . . . . . .

9 The system was verified by measuring the impedance $z$ of two different airterminated transmission lines. The theoretical prediction given by Eq. (7) is shown with solid lines and measurements are shown with open circles. Magnitude and phase for $d=13.98 \mathrm{~cm}$ appears in (a) and (b), and for $d=8.40 \mathrm{~cm}$ in (c) and (d). Only every sixth measurement data point is shown, so that the theoretical curves remain visible. . . . . . . . . . . . . 28

10 The measured phase and magnitude of the reflection coefficient of a water-air interface is shown. . . . . . . . . . . . . . . . .

11 Accuracy and precision of three impedance tube systems as expressed in the measurement of the reflection coefficient of a water-air interface. Open circles represent the average measurement values and the error bars represent the measurement ranges for the 5-9 kHz frequency range. The 1947 and 1983 data were obtained from Ref. 7 (p. 429) and Ref. 11 (p. 91), respectively.

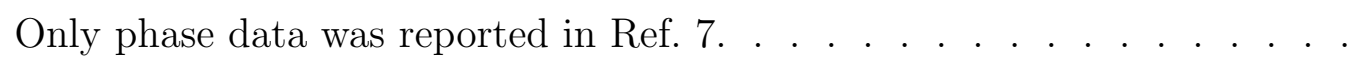




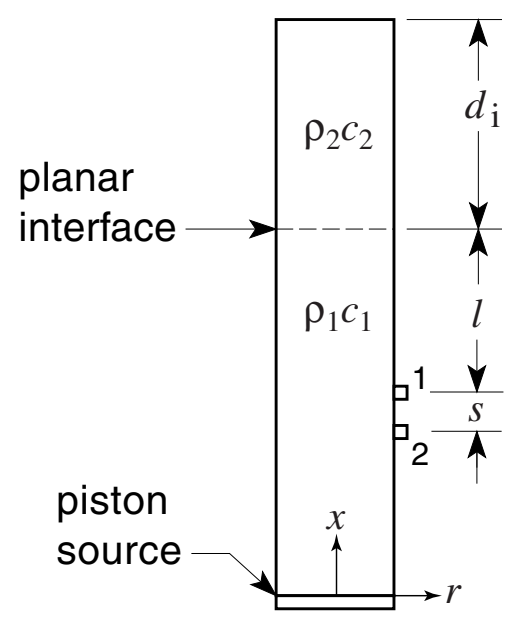

FIG. 1. 


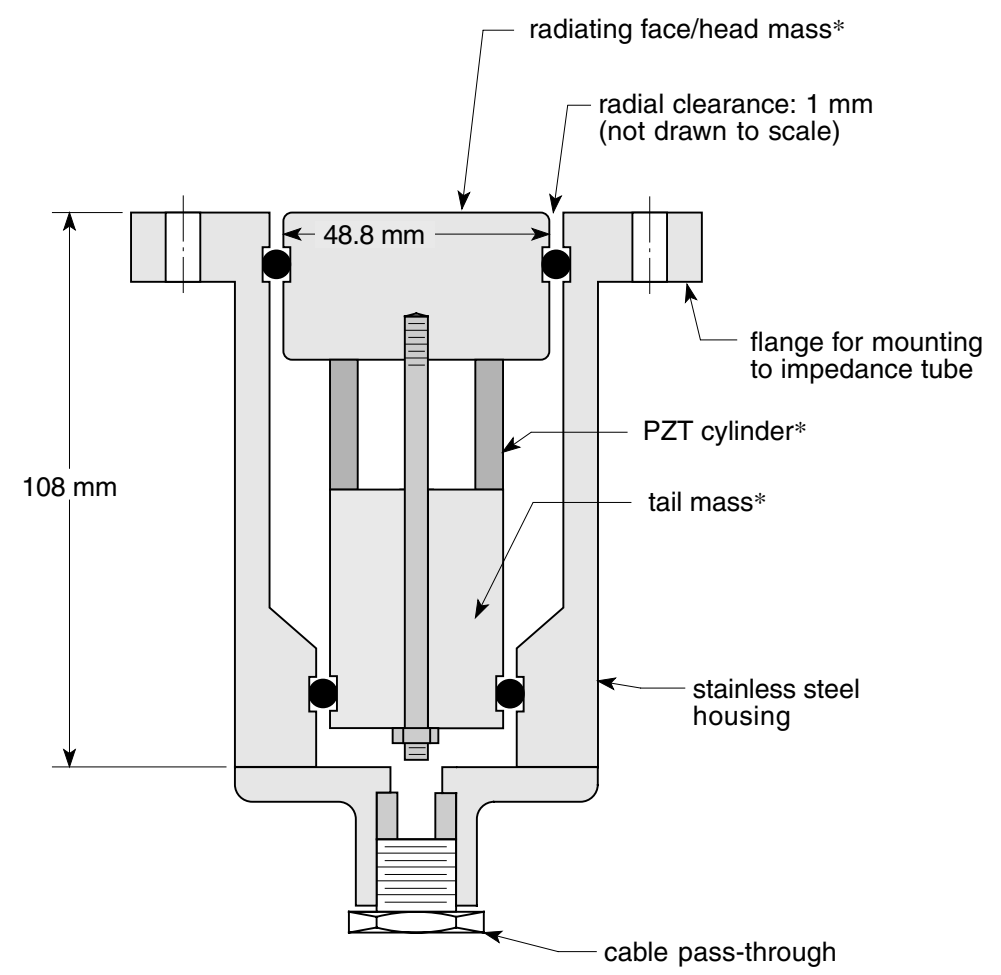

FIG. 2 . 


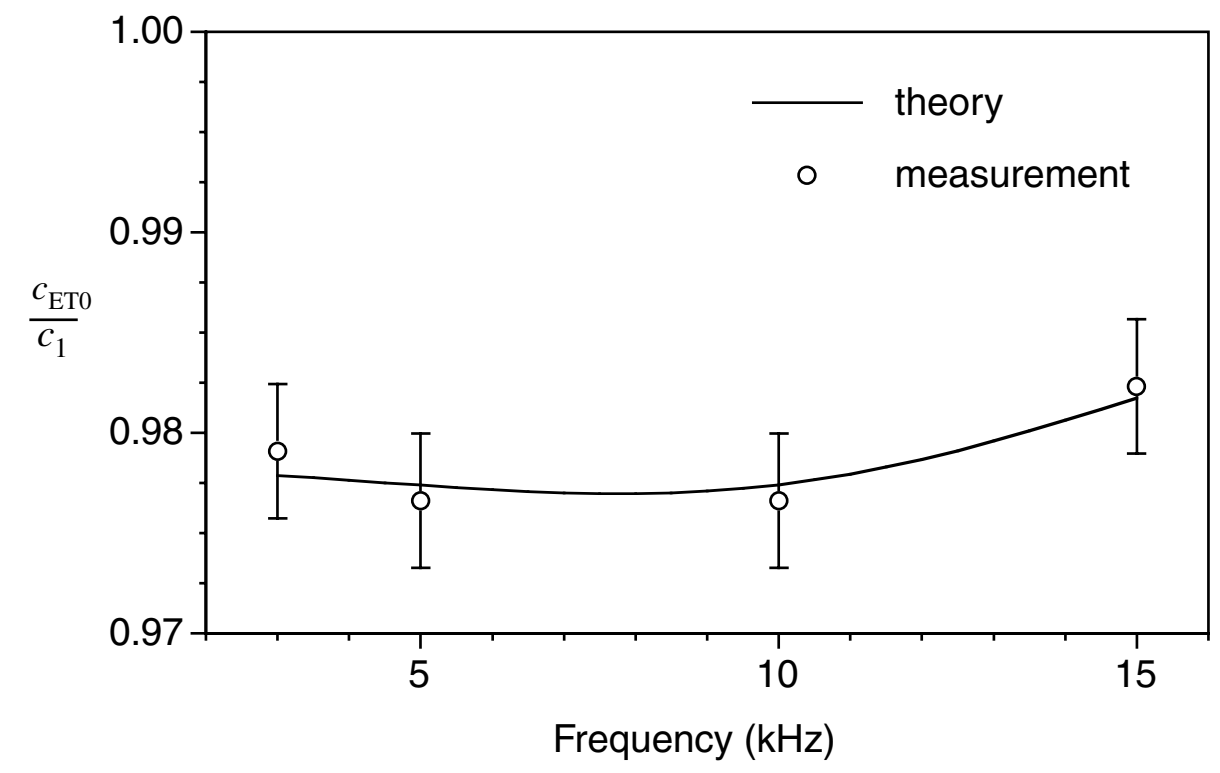

FIG. 3. 
(a)

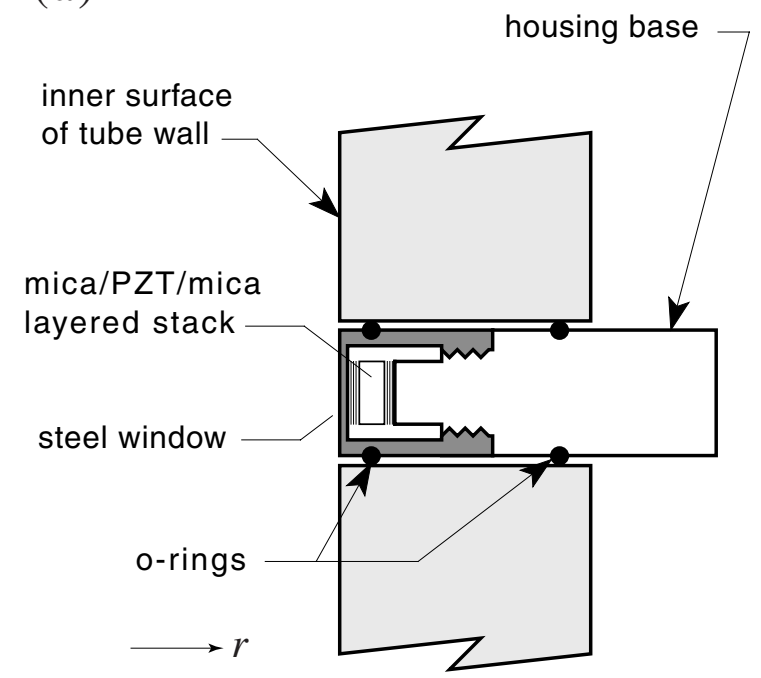

(b)

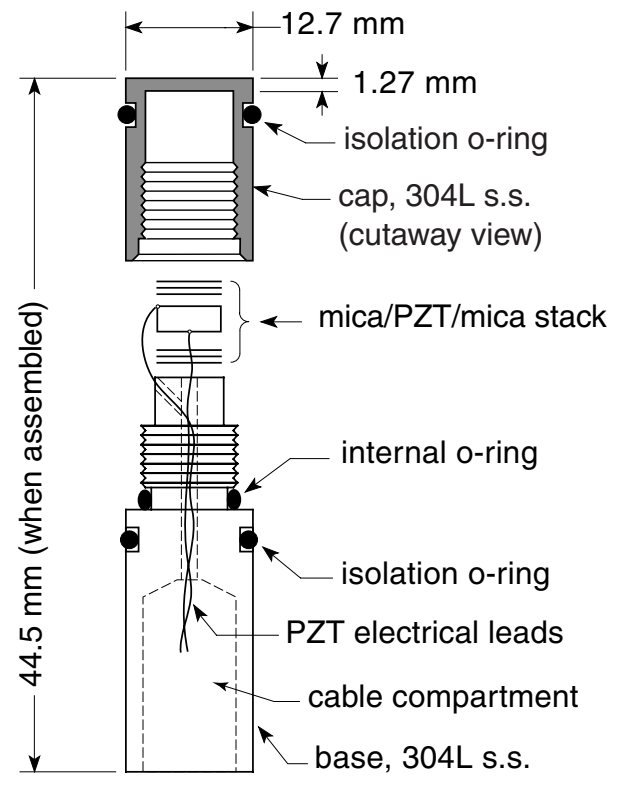

FIG. 4. 


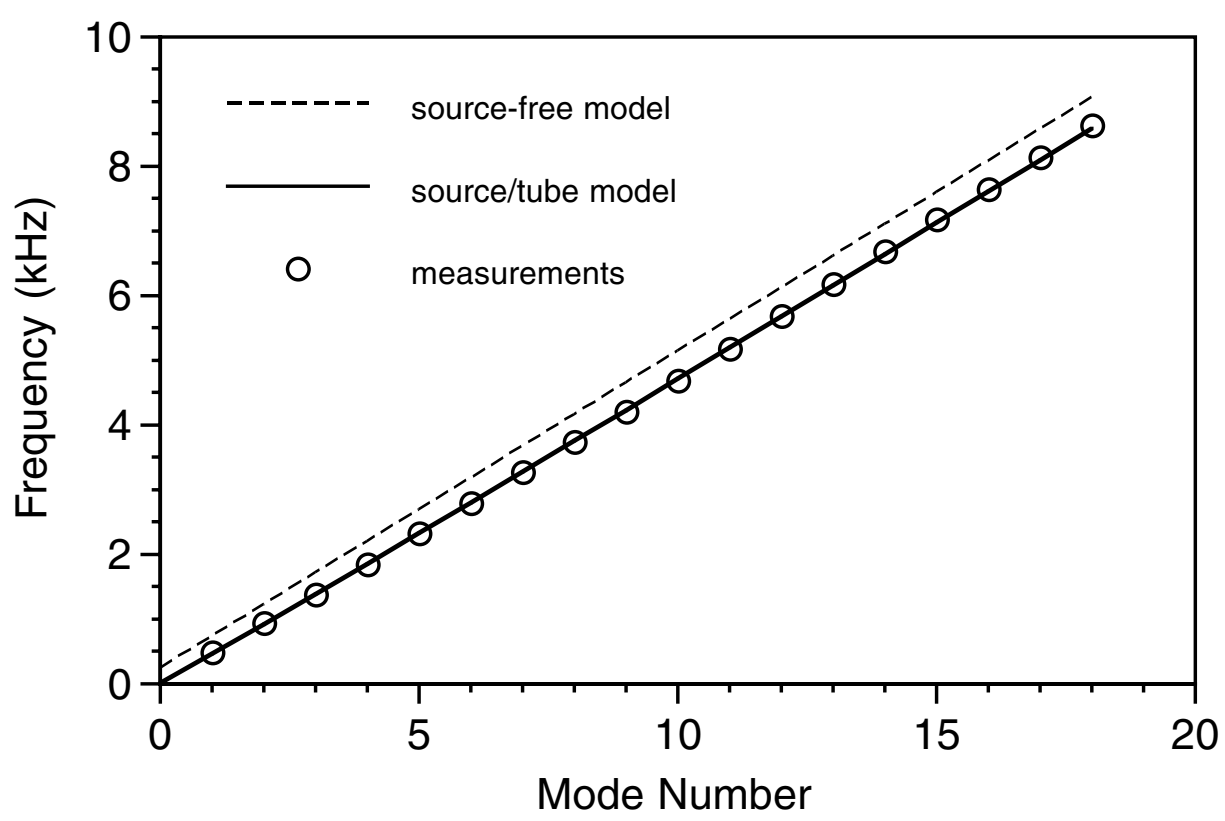

FIG. 5. 


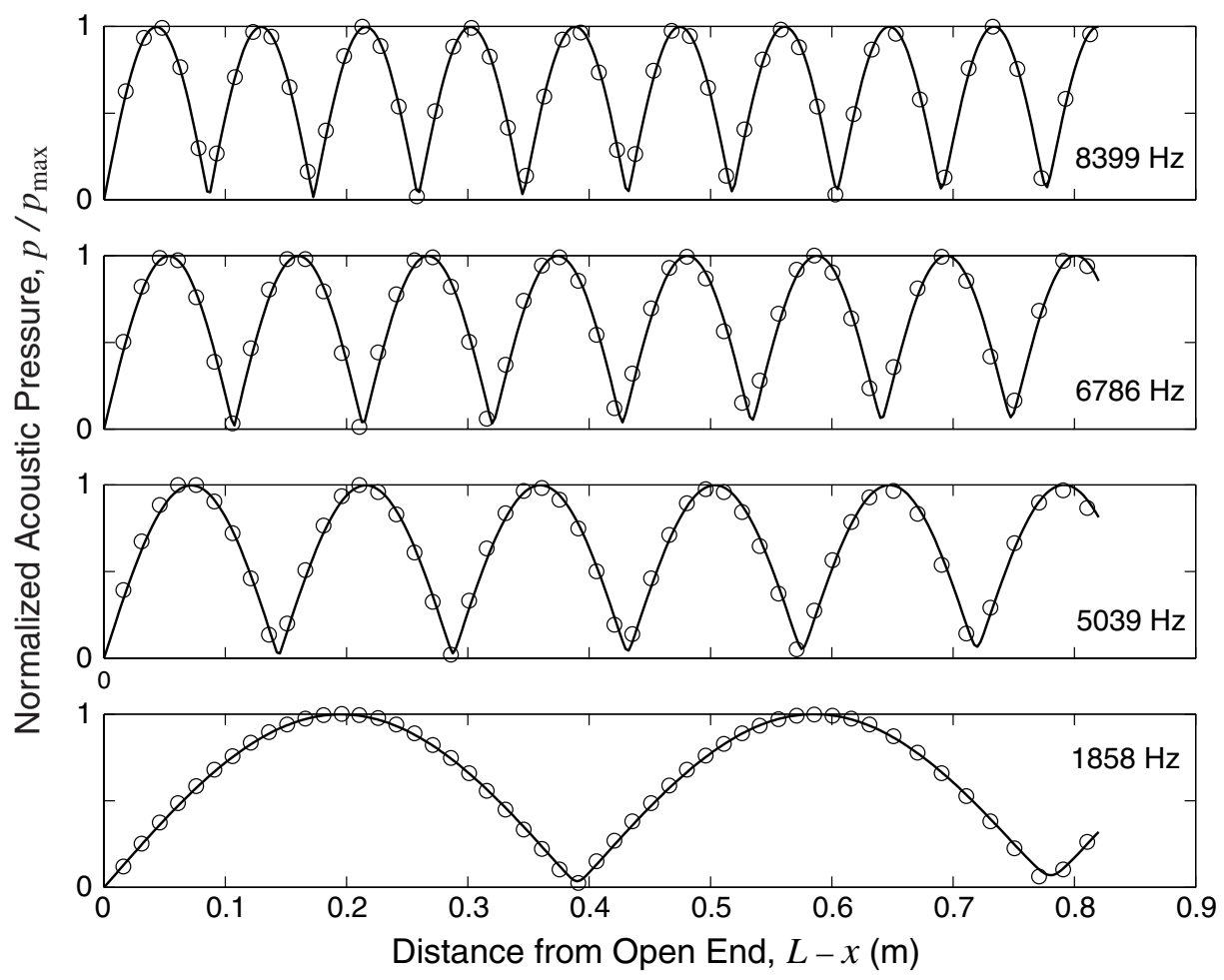

FIG. 6. 


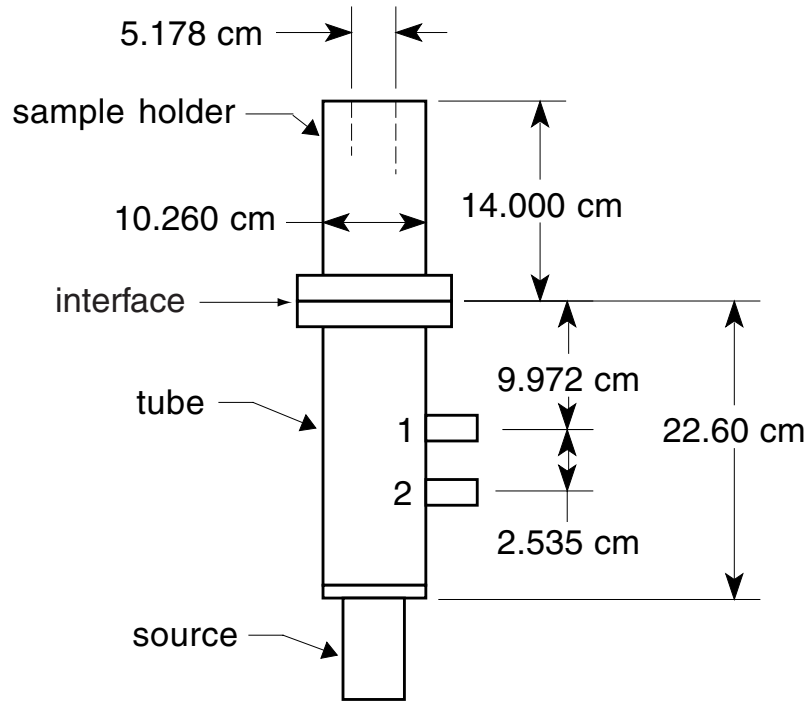

FIG. 7. 


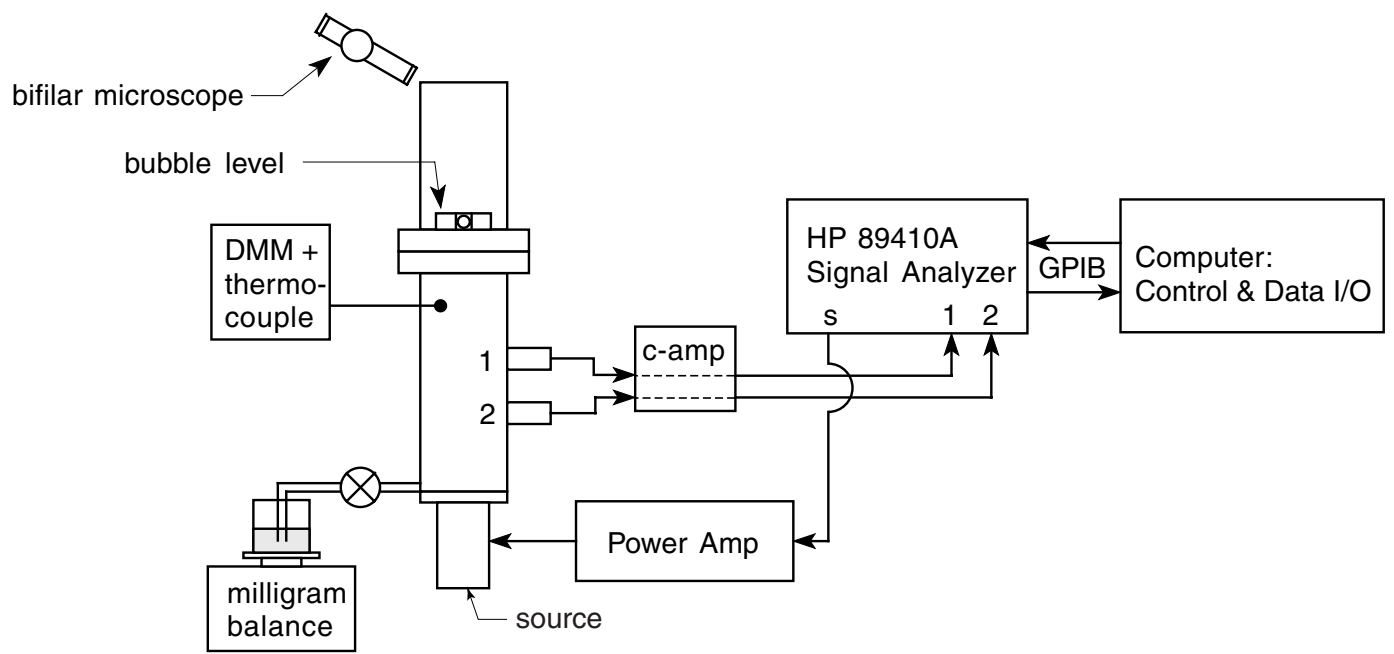

FIG. 8. 

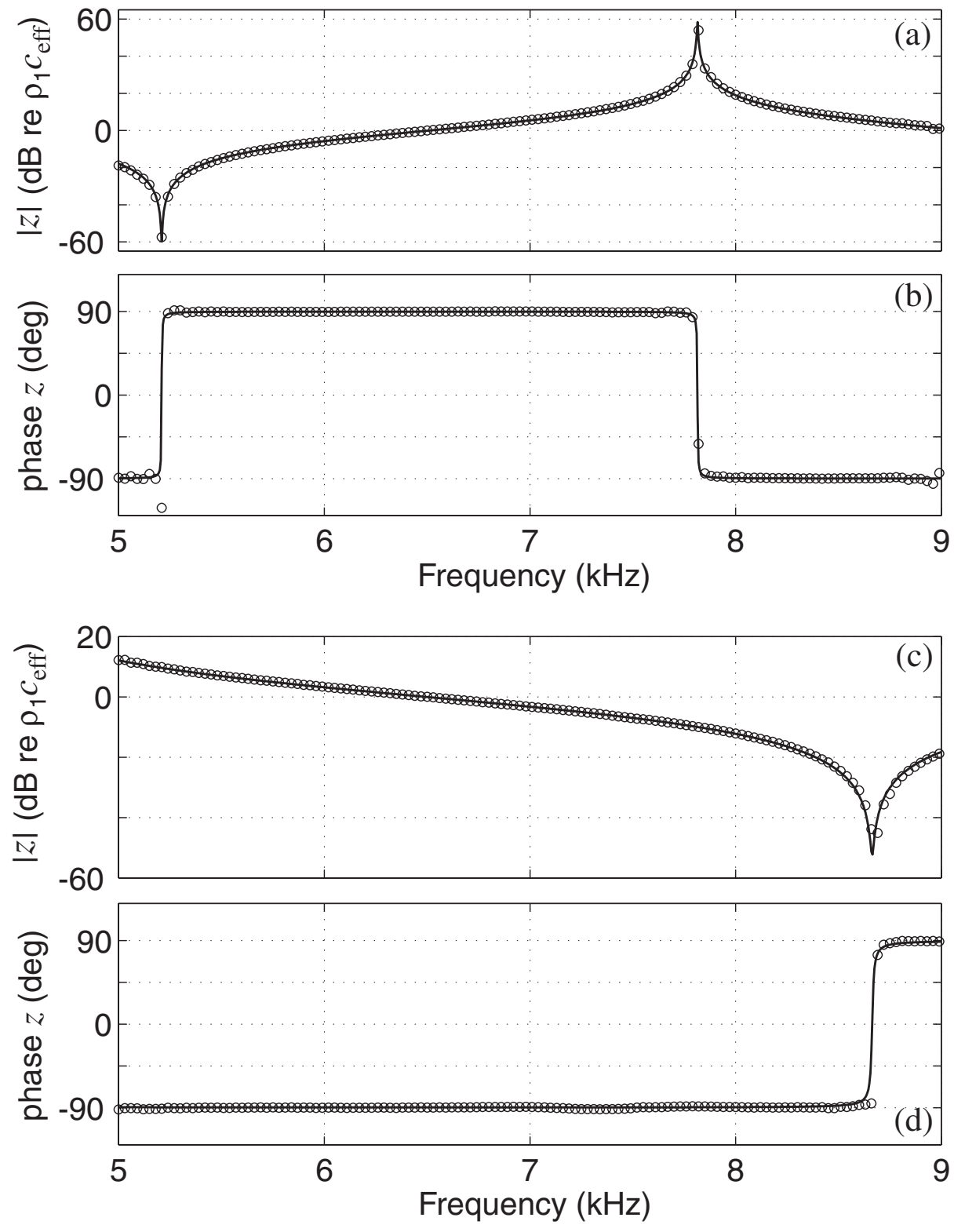

FIG. 9. 

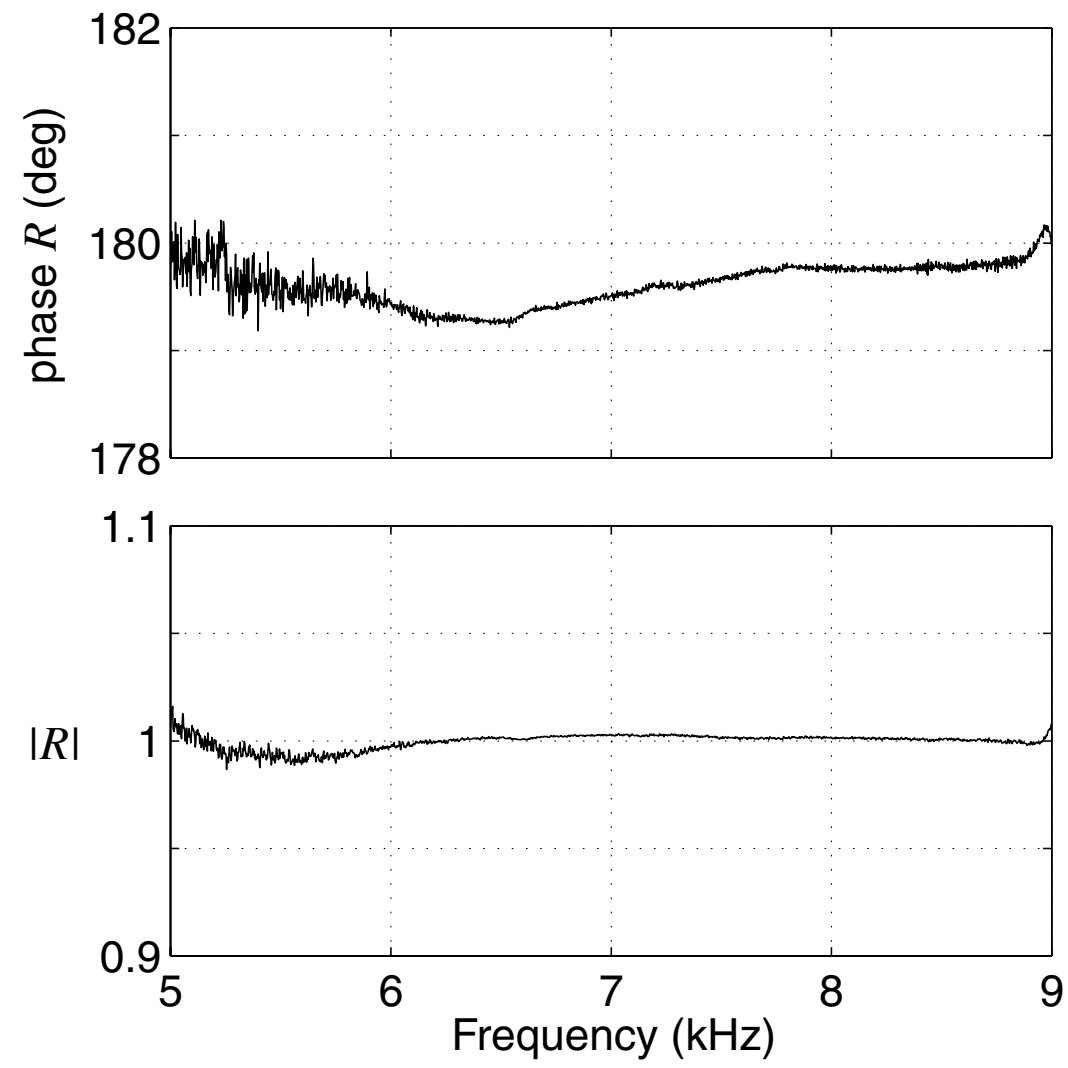

FIG. 10. 

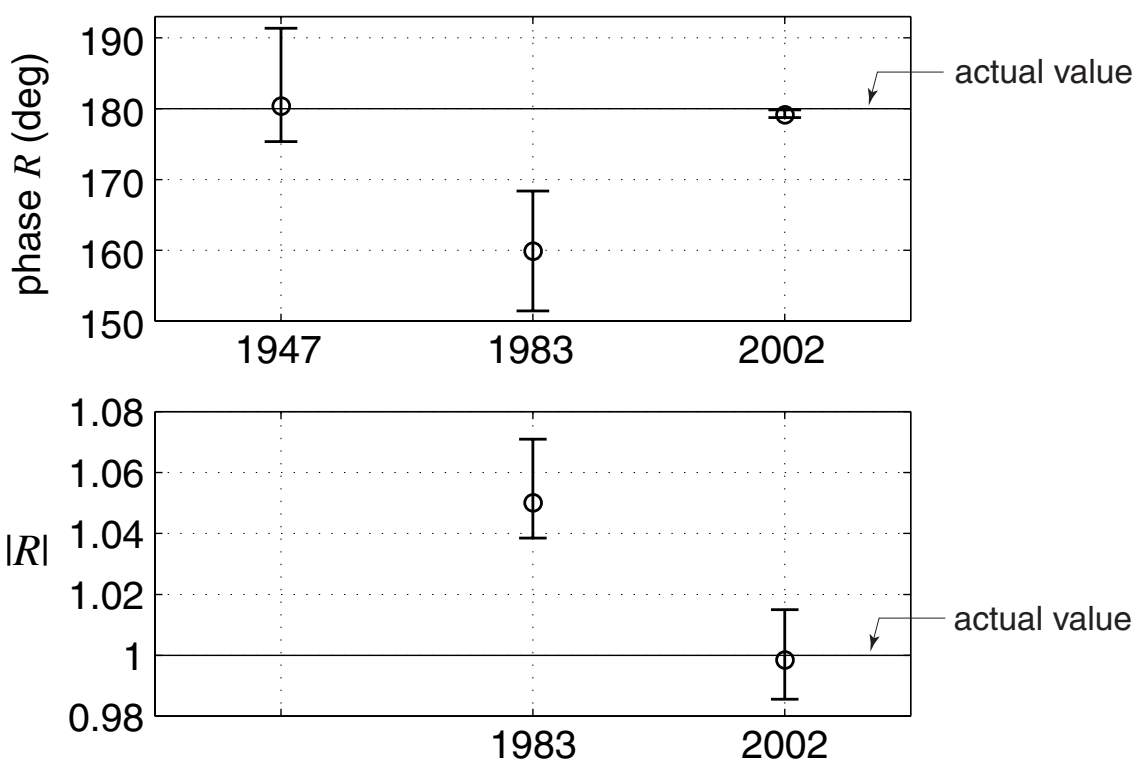

FIG. 11. 